\title{
Diosmetin and Its Glycoside, Diosmin, Improve Atopic Dermatitis- Like Lesions in 2,4-Dinitrochlorobenzene-Induced Murine Models
}

\author{
Sang-a Park ${ }^{1, \dagger}$, Sim-Kyu Bong ${ }^{1,2,}$, Jin Woo Lee ${ }^{1}$, No-June Park ${ }^{1}$, Yongsoo Choi ${ }^{1}$, Sang Moo Kim², \\ Min Hye Yang ${ }^{3}$, Yong Kee $\mathrm{Kim}^{4}$ and Su-Nam Kim ${ }^{1, *}$ \\ ${ }^{1}$ Natural Products Research Institute, Korea Institute of Science and Technology, Gangneung 25451, \\ ${ }^{2}$ Department of Marine Food Science and Technology, Gangneung-Wonju National University, Gangneung 25457, \\ ${ }^{3}$ College of Pharmacy, Pusan National University, Busan 46241, \\ ${ }^{4}$ College of Pharmacy, Sookmyung Women's University, Seoul 04610, Republic of Korea
}

\begin{abstract}
Naturally derived diosmetin and its glycoside diosmin are known to be effective in treating inflammatory disease. This study was performed to determine whether diosmin and diosmetin have the effect of improving atopic dermatitis in a 2,4-dinitrochlorobenzen (DNCB)-induced atopic dermatitis (AD) model. DNCB was used to establish AD model in hairless mice. Skin moisture, serum immunoglobulin $E(\mathrm{IgE})$, interleukin 4 (IL-4), and histological analysis were performed to measure the effectiveness of diosmin and diosmetine to improve AD. IL-4 levels were also measured in RBL-2H3 cells. Administration of diosmetin or diosmin orally inhibited the progress of DNCB-induced AD-like lesions in murine models by inhibiting transdermal water loss (TEWL) and increasing skin hydration. Diosmetin or diosmin treatment also reduced IgE and IL-4 levels in AD-induced hairless mouse serum samples. However, in the in vitro assay, only diosmetin, not diosmin, reduced the expression level of IL-4 mRNA in RBL-2H3 cells. Diosmin and diosmetine alleviated the altered epidermal thickness and immune cell infiltration in AD. Diosmin is considered effective in the cure of $A D$ and skin inflammatory diseases by being converted into diosmetin in the body by pharmacokinetic metabolism. Thus, oral administration of diosmetin and diosmin might be a useful agent for the treatment of AD and cutaneous inflammatory diseases.
\end{abstract}

Key Words: Diosmetin, Diosmin, Atopic dermatitis, IL-4, DNCB

\section{INTRODUCTION}

Atopic dermatitis (AD) is a chronic, recurrent allergic inflammatory skin disease characterized by itching, dry skin, and eczema (Wahlgren, 1999). It usually begins in infancy or childhood, and the distribution and response pattern of skin lesions vary slightly depending on the patient's age. In infants, acute eczema with exudates or scabs is common, often occurring on the face, head, and the outside of the limbs. In childhood between 2 and 10 years of age, rather than the face, it occurs in the folded parts of the limbs and the neck and often appears as a dry eczema. Atopic dermatitis often improves or disappears with age, and hand eczema often occurs in adulthood. When atopic dermatitis remains until adulthood, the skin symptoms of the body improve, but erythema, redness, and eczema tend to appear on the face. The folds are scratched for a long time, resulting in thickened skin with lichenification
(Spergel and Paller, 2003; Bieber, 2008).

The causes of atopic dermatitis are not yet known. Because clinical symptoms appear in various ways, such as dry skin and eczema, the causes of the disease cannot be explained in any one way. Thus, genetic predisposition, environmental factors, immunological reactions, and abnormalities of the skin's protective barrier function are considered to be the main causes of AD (David Boothe et al., 2017). In acute skin lesions of atopic dermatitis, Type 2 T helper cells (Th2) are present, and the cytokines interleukin 4 (IL-4), IL-5, and IL-13 are overexpressed, which not only causes allergic reactions, but also reduces innate immune responses (Lloyd and Snelgrove, 2018). There have been reports that cytokines produced by the Th2 immune response directly degrade barrier function, suggesting a new concept of the relationship between skin barrier abnormalities and immunological abnormalities (Brandt and Sivaprasad, 2011).

\section{Open Access https://doi.org/10.4062/biomolther.2020.135}

This is an Open Access article distributed under the terms of the Creative Commons Attribution Non-Commercial License (http://creativecommons.org/licenses/by-nc/4.0/) which permits unrestricted non-commercial use, distribution, and reproduction in any medium, provided the original work is properly cited.
Received Jul 31, 2020 Revised Aug 19, 2020 Accepted Aug 25, 2020

Published Online Sep 17, 2020

\section{*Corresponding Author}

E-mail: snkim@kist.re.kr

Tel: +82-33-650-3503, Fax: +82-33-650-3529

${ }^{\dagger}$ The first two authors contributed equally to this work. 
IL-4, a representative Th2 cytokine, promotes the class switch of B cells to increase immunoglobulin E ( $\lg E)$ synthesis, induce mast cell activation, eosinophil infiltration and differentiation, and induce type I hypersensitivity (Kumar et al., 2012; Gandhi et al., 2017). In addition, it is known that over-expression of IL-4 reduces the expression of filaggrin, involucrin, and loricrin, which are the major constituent proteins of the skin barrier, inhibits the recovery of ceramide, and also suppresses the expression of antibacterial peptides, leading to defects in the skin barrier (Howell et al., 2009; Sawada et al., 2012; Bao et al., 2017).

Impairment of skin barrier function is thought to be one of the important causes of atopic dermatitis. Defective skin barriers do not function properly as permeability barriers, allowing allergen penetration and moisture loss. In addition, the dermatitis caused by immunological abnormalities may impair the skin's barrier function, so blocking such a vicious cycle is one of the important tasks of AD treatment (Cork et al., 2009).

Medications such as corticosteroids, which have been primarily prescribed to treat $A D$, have many side effects and cannot be used in the long term (Hajar et al., 2015). Therefore, there is a need to develop a new concept of therapeutic material that can substitute for these or reduce the use of existing therapeutic agents, with the goal of fewer side effects for longterm use. Recently, dupilumab, a biological agent that functions as a IL-4R $\alpha$ blocker, has been approved for the treatment of adult patients with severe atopic dermatitis by the U.S. Food and Drug Administration and has been reported to have good therapeutic effect (Beck et al., 2014). Thus, it has been demonstrated that modulating IL-4 is a good target for treating atopic dermatitis.

Flavonoids are polyphenolic compounds that are naturally produced as secondary metabolites in plants such as citrus species (Nogata et al., 2006). Flavonoids are classified into flavones, flavanols, flavanons, isoflavones and anthocyanins, depending on their chemical structure. Flavonoids have been studied and are reported to have a variety of physiological activities, including antioxidant, anti-inflammatory, anti-diabetic, anti-aging, and antibacterial (Wang et al., 2018).

Diosmetin (3',5,7-trihydroxy-4'-methoxyflavone), the aglycone part of the flavonoid glycoside diosmin (3',5,7-trihydroxy4'-methoxyflavone-7-ramnoside), occurs naturally in citrus fruits. Pharmacologically, diosmetin is reported to exhibit anti-oxidant, anti-inflammatory, anti-bacterial and anti-cancer activities (Patel et al., 2013). Diosmin is broken down into its aglycone, diosmetin, by enzymes in the gut bacteria before it is absorbed from the gastrointestinal tract (Kanaze et al., 2004). Diosmetin also inhibits the expression of $\beta$-hexosaminidase, which induces an inflammatory response from RBL-2H3 cells and inhibits the production of IgE receptor-mediated IL-4 in in vitro experiments (Mastuda et al., 2002).

In this study, diosmetin reduced the expression of IL-4 in $\mathrm{RBL}-2 \mathrm{H} 3$ cells, but not diosmin. Thus, we tried to investigate the efficacy of diosmetin based on the anti-inflammatory activity to improve the $A D$ pathology via oral administration in an $A D$ animal model induced by 2,4-dinitrochlorobenzene (DNCB). In addition, since diosmin has the potential to convert diosmetin by intestinal bacteria, we evaluated whether diosmin will exhibit efficacy in vivo when administered orally in a DNCB model.

\section{MATERIALS AND METHODS}

\section{Chemicals}

Diosmetin and diosmin were purchased from ChemFaces (Wuhan, China), their purity was over 98\%. 2,4-Dinitrochlorobenzene (DNCB) was obtained from Sigma-Aldrich Co (St. Louis, MO, USA). DMEM (Dulbecco's Modified Eagle's Media) and FBS (Fetal Bovine Serum) were obtained from HyClone Laboratories, Inc (Logan, UT, USA). Penicillin-streptomycin solution and Fast SYBR ${ }^{\circledR}$ Green Master Mix were purchased from Life Technologies (Waltham, MA, USA). An RNeasy mini kit and a ImProm-II Reverse Transcription System kit were purchased from Qiagen (Valencia, CA, USA) and Promega (Madison, WI, USA), respectively.

\section{Animals}

Six-week-old female SKH-1 hairless mice were obtained from Orient Bio Inc.'s animal facility (Seongnam, Korea) and maintained in an air-conditioned animal room at a temperature of $25 \pm 5^{\circ} \mathrm{C}$ and $55 \pm 5 \%$ humidity. Mice were allowed to consume standard laboratory feed and water freely. All animal experiments were conducted in accordance with the Guide for the Care and Use of Laboratory Animals of the National Institutes of Health (NIH publication no. 85-23, revised 1996) and were approved by the KIST Institutional Animal Care and Use Committee (Certification no. KIST-2017-081, approval date September 25, 2017).

\section{Induction of AD-like skin lesions in mice by DNCB and diosmetin or diosmin application}

$\mathrm{AD}$ was induced on the backs of $\mathrm{SKH}-1$ hairless mice by applying DNCB dissolved in a solution of $99 \%$ of acetone in water and olive oil in a volume ratio of $3: 1$. During the first 7 days, $1 \%$ DNCB was applied into the dorsal skin of mice once a day. From the 8th day after rubbing DNCB, mice were challenged with $100 \mu \mathrm{L}$ of $0.1 \%$ DNCB every 3 days for an additional 2 weeks. During that same period, diosmetin $(5,10$ or 25 $\mathrm{mg} / \mathrm{Kg} /$ day) or diosmin (50 mg/Kg/day) dissolved in a solution of $2 \%$ carboxymethyl cellulose and water in a volume ratio of 3:2 was orally administered once a day. On the last day of the experiment, erythema, edema and lichenification were evaluated visually in hairless mice. Then, mice were sacrificed, and blood and skin tissue were collected for further studies.

\section{Measurement of transepidermal water loss (TEWL) and skin hydration}

To assess skin barrier status, TEWL was measured using an AquaFlux (Biox, London, UK), and hydration was measured using a Skin-O-Mat (COSMOMEP, Berlin, Germany). These two parameters measured weekly under $25 \pm 5^{\circ} \mathrm{C}$ and $55 \pm 5 \%$ humidity conditions.

\section{Histopathological examinations}

To evaluate histopathological variations, the back skin from SKH-1 hairless mice was fixed with $10 \%$ formalin and covered with paraffin wax. Sections were cut to $10 \mu \mathrm{m}$ using a microtome, placed on slides, then dried and stained with hematoxylin and eosin (H\&E) or toluidine blue. Histopathological examinations were performed using an Olympus CX31/ BX51 microscope (Olympus Optical Co., Tokyo, Japan), and photographs were obtained using a TE-2000U camera (Nikon Instruments Inc., Melville, NY, USA). The thicknesses of the 
epidermal layer were measured using a microscope equipped with a ruler and the LAS v4.8 (Leica Microsystem, Herbrugg, Switzerland) program.

\section{Determination of serum IgE and IL-4 concentrations}

Blood specimens were taken from the abdominal aortas of the mice and centrifuged at $10,000 \mathrm{rpm}$ for $15 \mathrm{~min}$ at $4^{\circ} \mathrm{C}$. Serum IgE and IL-4 concentrations were determined using ELISA kits (eBioscience, San Diego, CA, USA), according to the manufacturer's protocol.

\section{Cells and cell culture}

The RBL-2H3 cell line, a rat basophilic leukemia cell line, was purchased from ATCC (American Type Culture Collection, \#CRL-2256, Bethesda, MD, USA) and cultured in DMEM containing $10 \% \mathrm{FBS}$ and $1 \%$ penicillin-streptomycin in $\mathrm{a}_{2}$ incubator at $37^{\circ} \mathrm{C}$.

\section{Quantitative real-time PCR (Q-PCR)}

RBL-2H3 cells were collected for further studies. After lysis of the pelleted cells, total RNA was isolated using an RNeasy mini kit (Qiagen), and cDNA synthesis was performed using an ImProm-II reverse transcription system (Promega). Q-PCR (quantitative real-time PCR) to measure mRNA expression was performed using a 7500 Fast Real-Time PCR system (Applied Biosystems, Foster City, CA, USA) and Fast SYBR ${ }^{\circledR}$ Green Master Mix (Life Technologies). All reactions were repeated three times, and the data were analyzed using 7500 System SDS software version 1.3.1 (Applied Biosystems). The PCR primer sets were as follows: IL-4 (NM_021283), forward 5'-ACC TTG CTG TCA CCC TGT TC-3' and reverse 5'-TTG TGA GCG TGG ACTCAT TC-3'; $\beta$-actin (NM_007393), forward 5'-TCA TCA CCA TCG GCA ACG-3' and reverse 5'TTC CT GAT GTC CAC GTC GC-3'.

\section{Statistical analysis}

All quantitative data for this study were obtained through at least two independent experiments and are denoted as mean \pm standard deviation. Statistical analyses were carried out by a one-way analysis of variance (ANOVA) and Tukey's multiple comparisons post hoc analysis.

\section{RESULTS}

\section{Diosmetin ameliorates severity of the DNCB-induced AD mice}

To investigate the effects of diosmetin on atopic dermatitis, we orally administered diosmetin at concentrations of 5,10 or $25 \mathrm{mg} / \mathrm{kg}$ in DNCB-induced mouse models. Oral application of diosmetin for two weeks to hairless mice stimulated by DNCB effectively inhibited AD symptoms such as swelling, erythema, erosion and dryness (Fig. 1A). Skin barrier dysfunction, such as increases in TEWL and decreases in skin water contents, is usually detected before the development of AD. On the last day of the treatment, the TEWL value was increased in the DNCB-treated group $\left(76.5 \pm 4.8 \mathrm{~g} / \mathrm{m}^{2} / \mathrm{h}\right)$ compared to the nontreated control group $\left(22.5 \pm 1.2 \mathrm{~g} / \mathrm{m}^{2} / \mathrm{h}\right)$, and the TEWL value was decreased by approximately $47.7 \%$ in the DNCB and diosmetin co-treated groups $\left(38.9-40.5 \mathrm{~g} / \mathrm{m}^{2} / \mathrm{h}\right)$ compared to the DNCB-treated group (Fig. 1B). Skin hydration was significantly increased in the DNCB-diosmetin group (26.6-29.2\%) com-
A
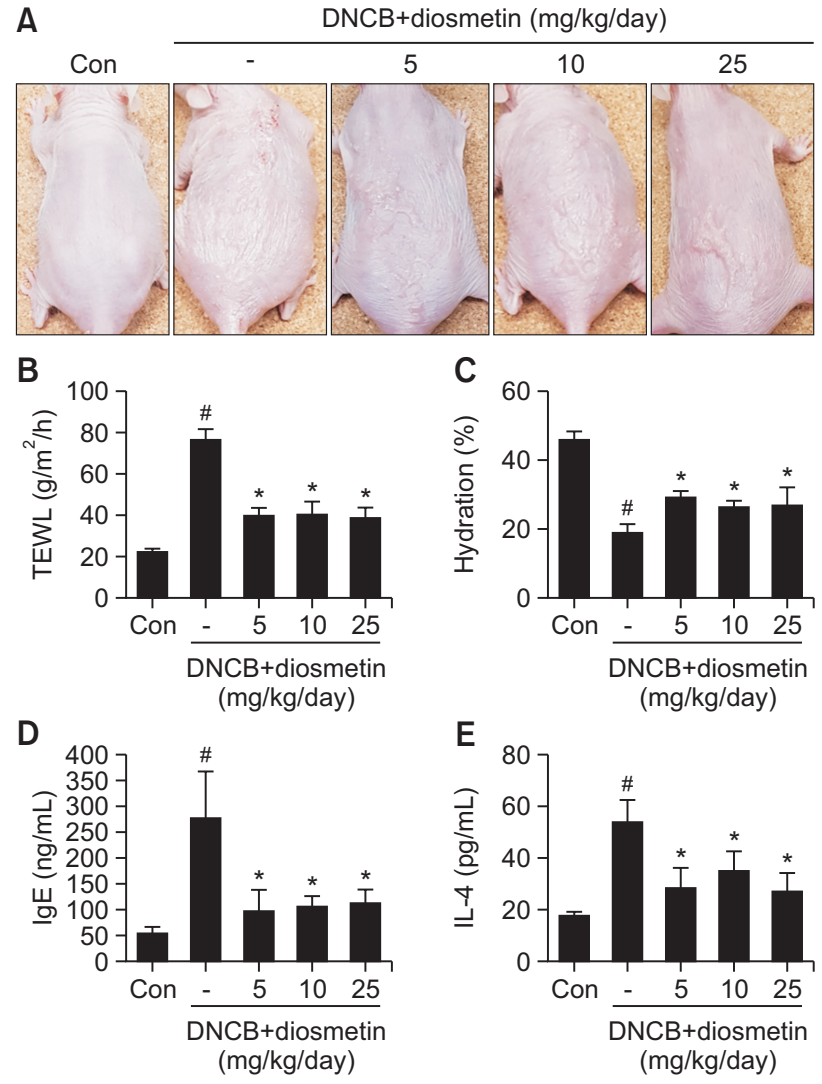

Fig. 1. Diosmetin ameliorated pathological changes in the skin of DNCB-induced atopic mice. Clinical features of AD-like skin lesions (A). Transepidermal water loss (TEWL) values (B). Level of skin hydration (C). Concentration of serum IgE (D). Concentration of serum IL-4 $(E)$. Data are presented as the mean \pm SD and were analyzed using a one-way ANOVA and Tukey's multiple comparisons post hoc analysis between the DNCB-induced group (DNCB) and the other groups (CON, DNCB-Diosmetin). ${ }^{*} p<0.05$ significantly greater or lower than $\mathrm{CON}$. ${ }^{*} p<0.05$ significantly greater or lower than DNCB. CON, normal control group; DNCB: DNCB-induced group, DNCB-Diosmetin: DNCB and Diosmetin-treated group.

pared to the DNCB group $(19.1 \pm 2.25)$ (Fig. 1C). Induction of atopic dermatitis by DNCB leads to strong increases in $\mathrm{lgE}$ $(278.4 \pm 86.9 \mathrm{ng} / \mathrm{mL})$ and IL-4 $(54.0 \pm 8.16 \mathrm{pg} / \mathrm{mL})$ concentrations in serum. However, application of diosmetin with DNCB reduced the serum levels of $\mathrm{IgE}(99.3-114.6 \mathrm{ng} / \mathrm{mL})$ and $\mathrm{IL}-4$ (27.1-35.2 pg/mL) in AD-induced hairless mouse models (Fig. 1D, 1E). There was a clear improvement in appearance with diosmetin administration, but it did not show significant differences in a dose-dependent manner. Taken together, our data showed that diosmetin improved AD-like symptoms and allergic responses in hairless mice.

\section{Diosmetin improves histological changes in the DNCB- induced AD mice}

The characteristic histological changes in patients with atopic dermatitis include very thick epidermis and dermis with large amounts of immune cells. On the last day of the experiment, to examine changes in the epidermis, the dorsal skin of the mice was separated and H\&E stained (Fig. 2A). In the DNCB-treated group $(45.6 \pm 3.5 \mu \mathrm{m})$, the epidermal 
thickness increased by $308 \%$ compared to the control group $(14.8 \pm 1.1 \mu \mathrm{m})$, and that of the diosmetin with DNCB-treated groups $(19.8-23.5 \mu \mathrm{m})$ decreased significantly compared to the DNCB-treated group (Fig. 2C). To confirm the degree of infiltration of immune cells in the dermis, the mouse tissues were stained with toluidine blue (Fig. 2B). Increased infiltration of inflammatory cells was detected in the DNCB-treated group (90 \pm 4/area), but diosmetin treatment diminished the number of cells to 49-62/area (Fig. 2D). Our data showed that diosme-
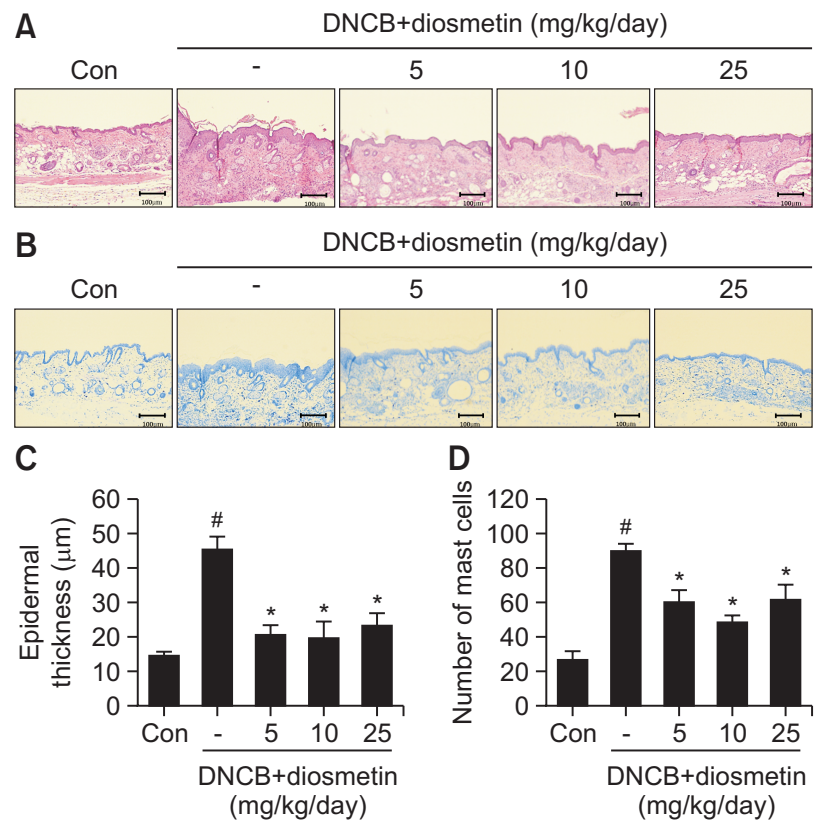

Fig. 2. Diosmetin improved histopathological changes, epidermal thickness and mast cell numbers in DNCB-induced AD-like symptoms in hairless mice. Hematoxylin and eosin staining (100x magnification, A). Toluidine blue staining (B). Epidermal thickness (C). Number of mast cells (D). Data are presented as the mean \pm SD and were analyzed using a one-way ANOVA and Tukey's multiple comparisons post hoc analysis between the DNCB-induced group (DNCB) and the other groups (CON, DNCB-Diosmetin). ${ }^{*} p<0.05$ significantly greater than $\mathrm{CON}$. ${ }^{*} p<0.05$ significantly lower than DNCB. CON, normal control group; DNCB: DNCB-induced group, DNCB-Diosmetin: DNCB and Diosmetin-treated group. tin could play a potent role in ameliorating $A D$.

\section{Diosmin, a glycoside compound of diosmetin, does not reduce IL-4 expression in RBL-2H3 cells}

Diosmin is a glycoside of diosmetin, which is metabolized in the body and converted to diosmetin (Fig. 3A). Diosmetin is known to inhibit the release of IL-4 in RBL-2H3 cells (Mastuda et al., 2002). Therefore, we decided to check whether diosmin could regulate the Th2 cytokine IL-4. As shown in Fig. 3B, the diosmetin-treated group exhibited reduced IL-4 expression compared to the PI-treated group, but diosmin did not affect the change in IL-4 expression. These data demonstrated that diosmin must be metabolized to diosmetin to show activity of IL-4 expression inhibition, and this metabolism does not occur at the cellular level.

\section{Diosmin diminishes severity of the DNCB-induced AD mice}

Next, we measured whether orally administering diosmin has an improvement effect on atopic dermatitis in DNCB-induced $A D$ mice. According to the data in the previous section, it was estimated that diosmin is metabolized to diosmetin and shows efficacy in vivo. Since the molecular weight of diosmin (608.5) is twice that of diosmetin (300.26), diosmin was orally administered to the mice at $50 \mathrm{mg} / \mathrm{kg}$, which is twice the highest concentration of diosmetin; this corresponds to almost the same molar concentration compared to diosmetine. The results showed that the group treated with diosmin and DNCB experienced the alleviation of several symptoms that were apparently seen on the skin surface in $A D$ compared to the group treated with DNCB only (Fig. 4A). TEWL values were measured on the last day of the experiment. In terms of TEWL value, the diosmin-treated group $(41.25 \pm 0.95 \mathrm{~g} /$ $\mathrm{m}^{2} / \mathrm{h}$ ) was reduced by approximately $30 \%$ compared to the DNCB-treated group (58.94 $\left.\pm 5.62 \mathrm{~g} / \mathrm{m}^{2} / \mathrm{h}\right)$ (Fig. 4B). The skin moisture content of the diosmin-administered group (28.3 \pm 5.44) was increased by approximately $61.7 \%$ compared to the DNCB-treated group (17.5 \pm 4.57$)$ (Fig. 4C). Levels of IgE $(218.46 \pm 37.98 \rightarrow 151.38 \pm 35.55)$ and IL-4 $(54 \pm 8.16 \rightarrow 28.7 \pm$ 7.25), which are indicators of atopic dermatitis in serum in the diosmin-treated group, also decreased by $30.7 \%$ and $46.9 \%$, respectively, compared to the DNCB-treated group (Fig. 4D, $4 \mathrm{E})$. Taken together, our study confirmed that diosmin improves all symptoms of $A D$ and blood index when administered orally.
A

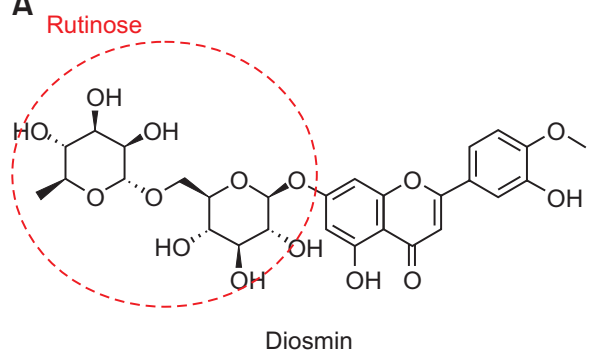

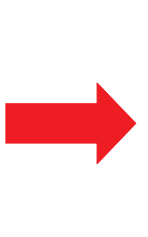<smiles>COc1ccc(-c2cc(=O)c3c(O)cc(O)cc3o2)cc1O</smiles>

Diosmetin

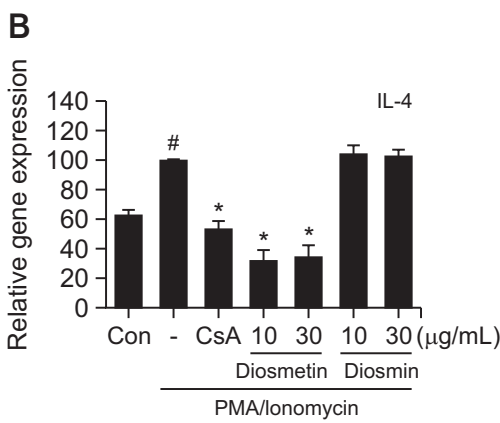

Fig. 3. Anti-allergic effects of diosmetin or diosmin in RBL-2H3 cells. Chemical structures of diosmin and diosmetin (A). IL-4 mRNA expression (B). Data are presented as the mean \pm SD and were analyzed using a one-way ANOVA and Tukey's multiple comparisons post hoc analysis between the PMA/lonomycin-treated group and the other groups. ${ }^{*} p<0.05$ significantly greater than $\mathrm{CON}$. ${ }^{*} p<0.05$ significantly lower than PMA/lonomycin-treated group. CON, normal control group; CsA, $1 \mu \mathrm{M}$ Cyclosporin A-treated group. 
A
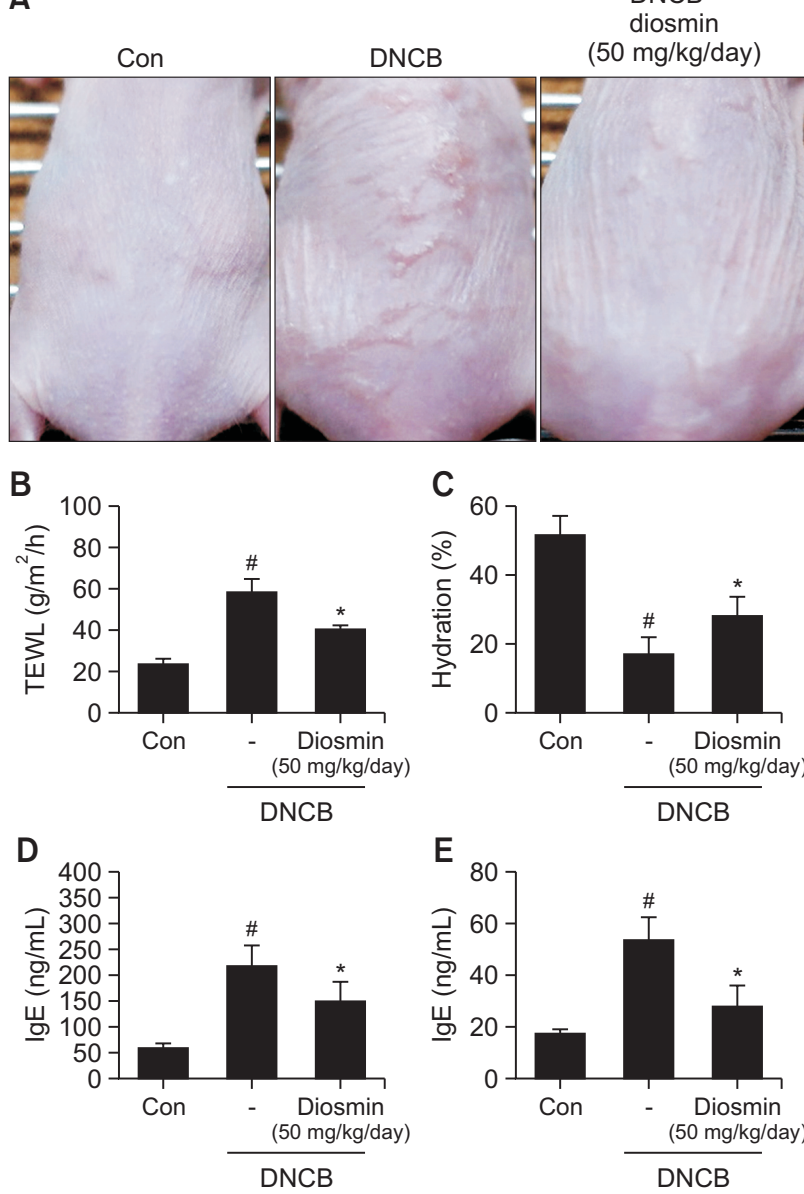

A

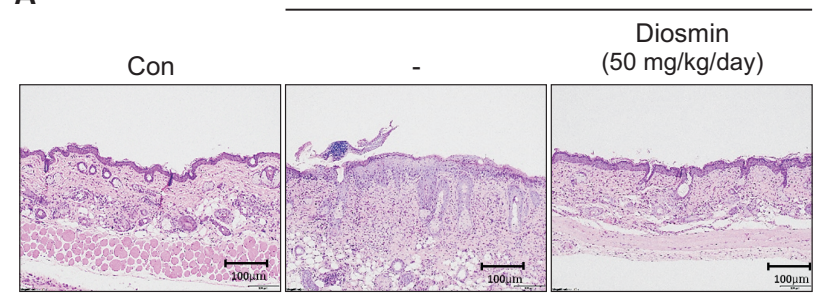

B

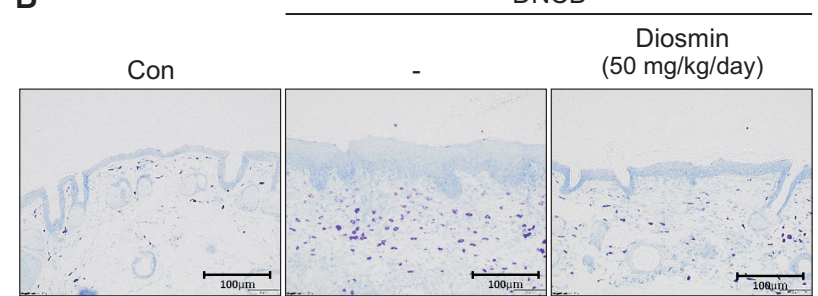

C
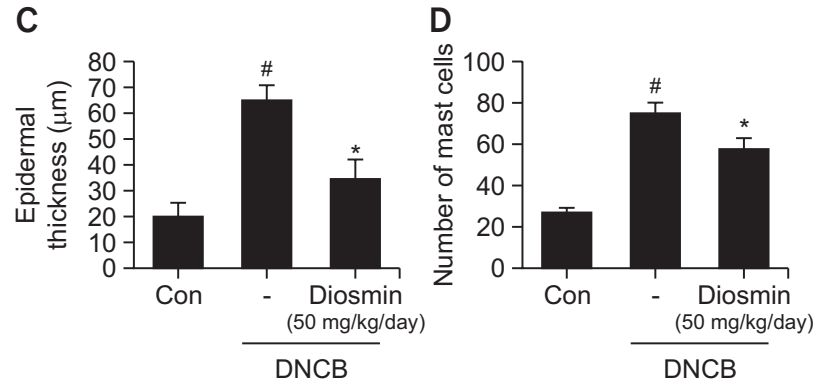

Fig. 5. Diosmin improved histopathological changes, epidermal thickness and mast cell numbers in DNCB-induced AD-like symptoms in hairless mice. Hematoxylin and eosin staining (100× magnification, A). Toluidine blue staining (B). Epidermal thickness (C). Number of mast cells (D). Data are presented as the mean \pm SD and were analyzed using a one-way ANOVA and Tukey's multiple comparisons post hoc analysis between the DNCB-induced group (DNCB) and the other groups (CON, DNCB-Diosmin). ${ }^{*} p<0.05$ significantly greater than $\mathrm{CON} .{ }^{*} p<0.05$ significantly lower than DNCB. CON, normal control group; DNCB: DNCB-induced group, DNCB-Diosmin: DNCB and Diosmin-treated group.

\section{DISCUSSION}

Naturally produced products can be a rich source of medicines. Substances derived from natural products have been widely used for skin-related problems because of their anti-inflammatory, anti-bacterial and dermatological efficacy. Therefore, research on natural products is one of the important arms of the development of new drugs to cure skin-derived diseases.

Atopic dermatitis is characterized by overexpression of Th2 cytokines and plasma IgE and itching of the outer skin. Patients with $A D$ generally have increased cytokines such as IL4, IL-5 and IL-13 via Th2 mediation (Liu et al., 2011). Among variable factors in chronic $A D$, in particular, IL-4 acts as an initiator of $A D$ and serves as the first mediator to trigger a series of subsequent atopic reactions. Therefore, we aimed to identify candidates for natural products that would be effective in treating $A D$ by inhibiting IL-4 expression.

Diosmin was first isolated from figwort (Scrophularia nodosa) and is a flavonoid compound that is mainly isolated 
from citrus peel (Campanero et al., 2010; Bogucka-Kocka et al., 2013). Diosmin has been widely used as a vasoprotective agent for venous vascular diseases, such as venous leg ulcers and hemorrhoids (Guilhou et al., 1997; Diana et al., 2000). In addition, since it has a strong anti-cancer effect, many researchers consider it as an alternative treatment for various cancers that occur in the liver, colon, and urinary system (Tanaka et al., 1997; Yang et al., 1997; Perumal et al., 2018). Antioxidant, anti-diabetic and anti-ulcer effects of diosmin have also been reported (Arab et al., 2015; Ahmed et al., 2016).

Diosmin, when administered orally, is rapidly hydrolyzed to flavone aglycone diosmetin by gut bacteria such as Clostridium, Lactobacillus, Bifidobacterium or Escherichia and is then absorbed in the intestine. Diosmin cultured with human gut microorganisms was rapidly converted to diosmetin (Campanero et al., 2010; Currò, 2018). In addition, as a result of detection in human plasma after oral administration of diosmetin to healthy volunteers, a small amount of its unconjugated form was detected (Campanero et al., 2010). Therefore, when evaluating the activity of diosmin, it is necessary to evaluate the activity of diosmetin. In addition, in vitro experiments have reported that only diosmetin is more effective than diosmin (Villa et al., 1992), which is in good agreement with our data on IL-4 expression.

To confirm whether diosmin and diosmetin have therapeutic efficacy against $A D$, hairless mice in which $A D$-like skin lesions were evoked by DNCB were used for in vivo studies. DNCB is a well-known hypersensitivity inducer that induces $A D$-like lesions in laboratory animals. Sensitization caused by DNCB induces symptoms resulting from the loss of skin barrier function, such as loss of percutaneous epidermal moisture and dry atopyic skin. The dorsal skin of diosmetin-treated hairless mice with induced AD-like skin lesions showed decreases in epidermal thickness and TEWL, but an increase in hydration of the skin.

We also investigated whether oral administration of diosmin can improve AD symptoms. Oral administration of diosmin to hairless mice with induced AD-like skin lesions with DNCB demonstrated that the impaired skin barrier function was restored, as evidenced by the improvement in changes in skin moisture seen in AD. Histological changes have shown that oral administration of diosmin accelerates recovery of the skin barrier and immune hyperactivation by exerting reduced effects on the thickness of the epidermis and the levels of invasive lymphocytes in the back skin.

The impairment of skin barrier function caused by skin sensitization is characterized by over-activated cytokines of helper T cells, leading to inflammatory skin disease. The major cytokine that regulates overproduced IgE synthesis is IL-4, which is considered an important biological marker of allergic diseases such as $A D$ and asthma. In this experiment, the levels of IgE and IL-4 in plasma were significantly increased in $D N C B$-induced AD hairless mice, and the levels were decreased in the diosmetin- and diosmin-treated groups.

In vitro, however, diosmin did not inhibit IL-4 mRNA overexpression in RBL-2H3 cells with PMA/ionomycin (PI), and only diosmetin inhibited the mRNA expression of the IL-4 gene. It is thought that orally administered diosmin is converted to diosmetin by pharmacokinetic metabolism in the body, which is effective in the treatment of $A D$ and skin inflammatory diseases.

In vivo experiments in $\mathrm{DNCB}$-induced $\mathrm{AD}$ hairless mice showed that oral administration of diosmetin improved AD. Administration of diosmetin improved skin barrier function and suppressed overexpression of plasma IgE and IL-4 in AD-like lesion animal models. In addition, the animal models administered orally with diosmin have been shown to improve AD. Diosmin's AD-improving effect in these in vivo experiments is thought to be evident, as diosmin is converted to diosmetin by pharmacokinetic metabolism and is absorbed into the body. Therefore, this study showed the effect of improving AD for diosmetin, one of the metabolites of diosmin, but further study for the effects of other metabolites of diosmin on $A D$ also is required.

\section{CONFLICT OF INTEREST}

The authors declare no conflict of interest.

\section{ACKNOWLEDGMENTS}

This work was supported by a grant from the Bio \& Medical Technology Development Program of the National Research Foundation (NRF) funded by the Ministry of Science \& ICT (grant Nos. NRF-2019M3A9I3080263, NRF2019M3A9I3080265, and NRF-2019M3A9I3080266) and the Korea Institute of Science \& Technology, Republic of Korea (Grant No. 2Z06260).

\section{REFERENCES}

Ahmed, S., Mundhe, N., Borgohain, M., Chowdhury, L., Kwatra, M., Bolshette, N., Ahmed, A. and Lahkar, M. (2016) Diosmin modulates the NF-kB signal transduction pathways and downregulation of various oxidative stress markers in alloxan-induced diabetic nephropathy. Inflammation 39, 1783-1797.

Arab, H. H., Salama, S. A., Omar, H. A., Arafa el, S. A. and Maghrabi, I. A. (2015) Diosmin protects against ethanol-induced gastric injury in rats: novel anti-ulcer actions. PLOS ONE 10, e0122417.

Bao, L., Mohan, G. C., Alexander, J. B., Doo, C., Shen, K., Bao, J. and Chan, L. S. (2017) A molecular mechanism for IL-4 suppression of loricrin transcription in epidermal keratinocytes: implication for atopic dermatitis pathogenesis. Innate Immun. 23, 641-647.

Beck, L. A., Thaçi, D., Hamilton, J. D., Graham, N. M., Bieber, T., Rocklin, R., Ming, J. E., Ren, H., Kao, R., Simpson, E., Ardeleanu, M., Weinstein, S. P., Pirozzi, G., Guttman-Yassky, E., Suárez-Fariñas, M., Hager, M. D., Stahl, N., Yancopoulos, G. D. and Radin, A. R. (2014) Dupilumab treatment in adults with moderate-to-severe atopic dermatitis. N. Engl. J. Med. 371, 130-139.

Bieber, T. (2008) Atopic dermatitis. N. Engl. J. Med. 358, 1483-1494.

Bogucka-Kocka, A., Woźniak, M., Feldo, M., Kockic, J. and Szewczyk, K. (2013) Diosmin--isolation techniques, determination in plant material and pharmaceutical formulations, and clinical use. Nat. Prod. Commun. 8, 545-550.

Brandt, E. B. and Sivaprasad, U. (2011) Th2 cytokines and atopic dermatitis. J. Clin. Cell. Immunol. 2, 110.

Campanero, M. A., Escolar, M., Perez, G., Garcia-Quetglas, E., Sadaba, B. and Azanza, J. R. (2010) Simultaneous determination of diosmin and diosmetin in human plasma by ion trap liquid chromatography-atmospheric pressure chemical ionization tandem mass spectrometry: application to a clinical pharmacokinetic study. J. Pharm. Biomed. Anal. 51, 875-881.

Cork, M. J., Danby, S. G., Vasilopoulos, Y., Hadgraft, J., Lane, M. E., Moustafa, M., Guy, R. H., Macgowan, A. L., Tazi-Ahnini, R. and Ward, S. J. (2009) Epidermal barrier dysfunction in atopic dermatitis. J. Invest. Dermatol. 129, 1892-1908. 
Currò, D. (2018) The role of gut microbiota in the modulation of drug action: a focus on some clinically significant issues. Expert Rev. Clin. Pharmacol. 11, 171-183.

David Boothe, W., Tarbox, J. A. and Tarbox, M. B. (2017) Atopic dermatitis: pathophysiology. Adv. Exp. Med. Biol. 1027, 21-37.

Diana, G., Catanzaro, M., Ferrara, A. and Ferrari, P. (2000) Activity of purified diosmin in the treatment of hemorrhoids. Clin. Ter. 151, 341-344.

Gandhi, N. A., Pirozzi, G. and Graham, N. M. H. (2017) Commonality of the IL-4/IL-13 pathway in atopic diseases. Expert Rev. Clin. Immunol. 13, 425-437.

Guilhou, J. J., Dereure, O., Marzin, L., Ouvry, P., Zuccarelli, F., Debure, C., Van Landuyt, H., Gillet-Terver, M. N., Guillot, B., Levesque, H., Mignot, J., Pillion, G., Février, B. and Dubeaux, D. (1997) Efficacy of Daflon 500 mg in venous leg ulcer healing: a double-blind, randomized, controlled versus placebo trial in 107 patients. Angiology 48, 77-85.

Hajar, T., Leshem, Y. A., Hanifin, J. M., Nedorost, S. T., Lio, P. A., Paller, A. S., Block, J. and Simpson, E. L.; (the National Eczema Association Task Force) (2015) A systematic review of topical corticosteroid withdrawal ("steroid addiction") in patients with atopic dermatitis and other dermatoses. J. Am. Acad. Dermatol. 72, 541-549.e2.

Howell, M. D., Kim, B. E., Gao, P., Grant, A. V., Boguniewicz, M., DeBenedetto, A., Schneider, L., Beck, L. A., Barnes, K. C. and Leung, D. Y. (2009) Cytokine modulation of atopic dermatitis filaggrin skin expression. J. Allergy Clin. Immunol. 124, R7-R12.

Kanaze, F. I., Bounartzi, M. I. and Niopas, I. (2004) A validated HPLC determination of the flavone aglycone diosmetin in human plasma. Biomed. Chromatogr. 18, 800-804.

Kumar, S., Khandpu, S., Rao, D. N., Wahaab, S. and Khanna, N. (2012) Immunological response to Parthenium hysterophorus in Indian patients with Parthenium sensitive atopic dermatitis. Immunol. Invest. 41, 75-86.

Liu, F. T., Goodarzi, H. and Chen, H. Y. (2011) lgE, mast cells, and eosinophils in atopic dermatitis. Clin. Rev. Allergy Immunol. 41, 298-310.

Lloyd, C. M. and Snelgrove, R. J. (2018) Type 2 immunity: expanding our view. Sci. Immunol. 3, eaat1604.

Mastuda, H., Morikawa, T., Ueda, K., Managi, H. and Yoshikawa, M.
(2002) Structural requirements of flavonoids for inhibition of antigen-Induced degranulation, TNF-alpha and IL-4 production from RBL-2H3 cells. Bioorg. Med. Chem. 10, 3123-3128.

Nogata, Y., Sakamoto, K., Shiratsuchi, H., Ishii, T., Yano, M. and Ohta, H. (2006) Flavonoid composition of fruit tissues of citrus species. Biosci. Biotechnol. Biochem. 70, 178-192.

Patel, K., Gadewar, M., Tahilyani, V. and Patel, D. K. (2013) A review on pharmacological and analytical aspects of diosmetin: a concise report. Chin. J. Integr. Med. 19, 792-800.

Perumal, S., Langeshwaran, K., Selvaraj, J., Ponnulakshmi, R., Shyamaladevi, B. and Balasubramanian, M. P. (2018) Effect of diosmin on apoptotic signaling molecules in $\mathrm{N}$-nitrosodiethylamine-induced hepatocellular carcinoma in experimental rats. Mol. Cell. Biochem. 449, 27-37.

Sawada, E., Yoshida, N., Sugiura, A. and Imokawa, G. (2012) Th1 cytokines accentuate but Th2 cytokines attenuate ceramide production in the stratum corneum of human epidermal equivalents: an implication for the disrupted barrier mechanism in atopic dermatitis. J. Dermatol. Sci. 68, 25-35.

Spergel, J. M. and Paller, A. S. (2003) Atopic dermatitis and the atopic march. J. Allergy Clin. Immunol. 112, S118-S127.

Tanaka, T., Makita, H., Kawabata, K., Mori, H., Kakumoto, M., Satoh, K., Hara, A., Sumida, T., Tanaka, T. and Ogawa, H. (1997) Chemoprevention of azoxymethane-induced rat colon carcinogenesis by the naturally occurring flavonoids, diosmin and hesperidin. Carcinogenesis 18, 957-965.

Villa, P., Cova, D., De Francesco, L., Guaitani, A., Palladini, G. and Perego, R. (1992) Protective effect of diosmetin on in vitro cell membrane damage and oxidative stress in cultured rat hepatocytes. Toxicology 73, 179-189.

Wahlgren, C. F. (1999) Itch and atopic dermatitis: an overview. J. Dermatol. 26, 770-779.

Wang, T. Y., Li, Q. and Bi, K. S. (2018) Bioactive flavonoids in medicinal plants: structure, activity and biological fate. Asian J. Pharm. Sci. 13, 12-23.

Yang, M., Tanaka, T., Hirose, Y., Deguchi, T., Mori, H. and Kawada, Y. (1997) Chemopreventive effects of diosmin and hesperidin on $\mathrm{N}$ butyl-N-(4-hydroxybutyl)nitrosamine-induced urinary-bladder carcinogenesis in male ICR mice. Int. J. Cancer 73, 719-724. 